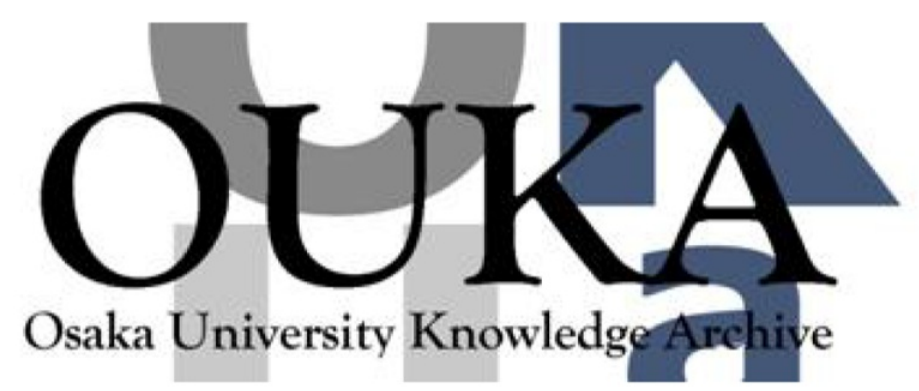

\begin{tabular}{|c|l|}
\hline Title & $\begin{array}{l}\text { Chain Stiffness of Cellulose } \\
\text { Tris(phenylcarbamate) in Tricresyl Phosphate } \\
\text { (TCP) }\end{array}$ \\
\hline Author(s) & Jiang, XinYue; Sato, Takahiro; Terao, Ken \\
\hline Citation & Polymer Bulletin. 75(3) p. 1265-p. 1273 \\
\hline Issue Date & $2018-03$ \\
\hline oaire:version & AM \\
\hline URL & https://hdl.handle. net/11094/71463 \\
\hline rights & \\
\hline Note & \\
\hline
\end{tabular}

Osaka University Knowledge Archive : OUKA

https://ir. Library. osaka-u. ac. jp/

Osaka University 


\title{
Chain stiffness of cellulose tris(phenylcarbamate) in tricresyl phosphate
}

\section{(TCP)}

\author{
Xin Yue Jiang, ${ }^{1}$ Takahiro Sato, ${ }^{1}$ and Ken Terao ${ }^{1}$
}

1 Department of Macromolecular Science, Graduate School of Science, Osaka University, 1-1 Machikaneyama-cho, Toyonaka, Osaka 560-0043, Japan.

Corresponding author: Ken Terao

E-mail: kterao@chem.sci.osaka-u.ac.jp

Phone: +81-6-6850-5459

ORCID: 0000-0001-7363-4491

\begin{abstract}
Small-angle X-ray scattering (SAXS) measurements were carried out for two cellulose tris(phenylcarbamate) (CTPC) samples in tricresyl phosphate (TCP) at $25{ }^{\circ} \mathrm{C}$ to determine the particle scattering function $P(q)$ and the $z$-average mean-square radius of gyration $\left\langle S^{2}\right\rangle_{z}$. The obtained data were analyzed in terms of the wormlike chain model to estimate the Kuhn segment length $\lambda^{-1}$ (the stiffness parameter, equivalent to twice the persistence length) and the helix pitch (or helix rise) per residue $h$. The resultant $\lambda^{-1}$ and $h$ were $11.5 \pm 0.5 \mathrm{~nm}$ and $0.51 \mathrm{~nm}$, respectively. While the latter value $(h)$ is consistent with the previously reported values both for cellulose and cellulose derivatives, appreciably higher chain flexibility was found for CTPC in TCP than that in tetrahydrofuran at $25^{\circ} \mathrm{C}(19-24 \mathrm{~nm})$. The value is fairly close to that in anisol, cyclohexanol, and benzophenone assuming an appropriate temperature coefficient. We may thus conclude that CTPC behaves as a semiflexible chain in TCP.
\end{abstract}




\section{Introduction}

Since cellulose tris(phenylcarbamate) (CTPC) of which chemical structure is illustrated in Fig. 1 is easily synthesized from natural cellulose [1,2] and soluble in many common organic solvents including theta solvents [3], much work has been done for dilute solution properties to elucidate the conformational characteristics of cellulosic chains [1,4-13]. Almost all reports indicate that CTPC behaves as a semiflexible polymer in solution. Although the conformational properties and intermolecular interactions of semiflexible polymers have been widely investigated [14-17], a comprehensive study through rheological and conformational properties is not still enough to elucidate the relationship including cellulose [18,19]. This is likely because suitable solvents for dilute solution properties are different from those for rheological properties, namely, low viscous and volatile organic solvents were favorably used to determine their conformation in solution, whereas highly viscous solvents with low vapor pressure are suitable to characterize polymer dynamics. As a preliminary study of this research, we found that CTPC is well soluble in tricresyl phosphate (TCP, Fig. 1), which is a useful solvent to investigate the rheological properties and indeed the dynamic birefringence and viscoelasticity of polystyrene in this solvent have been investigated in detail [20]. As a step of the comprehensive work of static and dynamic properties of semiflexible polymers in solution, we report the dimensional properties for CTPC in TCP in this paper.

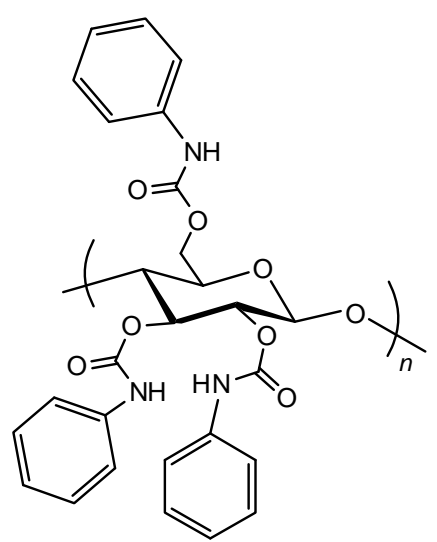

CTPC

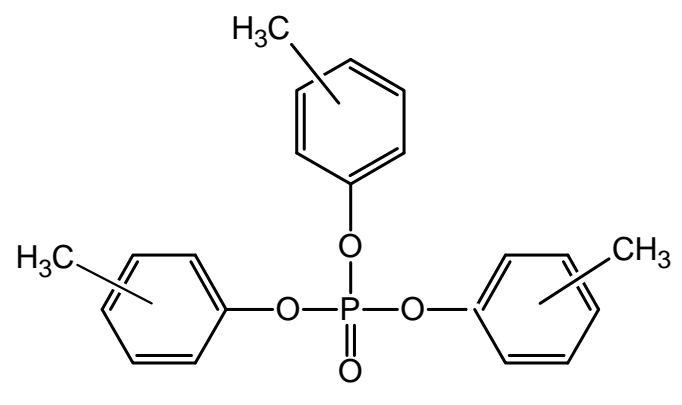

TCP

Fig. 1 Chemical structures of cellulose tris(phenylcarbamate) (CTPC) and tricresyl phosphate (TCP)

Regarding the chain stiffness of CTPC in solution, Dañhelka et al. [10] analyzed their own and older data $[4,7,9,8]$ to determine the chain stiffness parameter $\lambda^{-1}$ (Kuhn segment length or twice persistence length) of the Kratky-Porod wormlike chain [21] in different solvent conditions. While $\lambda^{-1}$ for CTPC in tetrahydrofuran (THF), acetone, dioxane, and pyridine at the temperatures $T$ of 20 or $25^{\circ} \mathrm{C}$ was reported to be in a rather narrow range from 19 to $27 \mathrm{~nm}$, the parameter in benzophenone, cycohexanol, and anisol was much smaller, that is, $7-10 \mathrm{~nm}$ at relatively high temperatures $\left(73-94{ }^{\circ} \mathrm{C}\right)$. Even though we consider the known temperature dependence of CTPC in 
four different solvents, that is, $\mathrm{d} \ln \lambda^{-1} / \mathrm{d} T \sim-6.5 \times 10^{-3} \mathrm{~K}^{-1}[13,22]$, the chain stiffness in the latter three solvents was found to be smaller than those in the former solvents. The solvent dependent conformation suggests that the intramolecular hydrogen bonding interactions and/or polymer-solvent interactions play an important role to determine the conformation of CTPC in dilute solution as pointed out by Sutter et al. [9]. We also showed that local helical structure stabilized by the intramolecular hydrogen bonds between the carbamate groups on the neighboring repeat units stiffen the main chain of cellulose tris(alkylcarbamate)s [23], curdlan tris(phenylcarbamate) [24], and amylose tris(alkylcarbamate)s [25-28] and furthermore hydrogen bonding solvent molecules significantly extend and stiffen the main chain of amylose tris(phenylcarbamate) and amylose tris(3,5-dimethylphenylcarbamate) [29,30]. The chain conformation of CTPC in arbitrary solvent cannot therefore be predicted without determining it directly in solution.

We thus made small-angle X-ray scattering (SAXS) measurements for two CTPC samples in TCP to determine the chain stiffness parameter because conventional light scattering measurements are not suitable for this purpose because of the difficulty of optical clean and very small contrast factor of visible light. Indeed, the dimensional properties of polystyrene in TCP were investigated by means of neutron scattering [31] and those for polysaccharides in an ionic liquid were studied by SAXS [19] due to the same reason.

\section{Experimental}

\section{Samples and test solutions}

Two CTPC samples stored in the laboratory originally synthesized and fractionated for the former study [11] were chosen and designated to be CTPC61K and CTPC84K. Their weight-average molar mass $M_{\mathrm{w}}$ and the dispersity index $Ð$ defined as the ratio of $M_{\mathrm{w}}$ to the number-average molar mass were determined to be $M_{\mathrm{w}}=6.08 \times 10^{4} \mathrm{~g}$ $\mathrm{mol}^{-1}$ and $Ð=1.1$ for CTPC61K and $M_{\mathrm{w}}=8.41 \times 10^{4} \mathrm{~g} \mathrm{~mol}^{-1}$ and $Ð=1.1$ for CTPC $84 \mathrm{~K}$ from a size-exclusion chromatography equipped with a multi-angle light scattering detector (SEC-MALLS) in THF. The details of the measurement were as reported elsewhere [23] and the literature value [11] of the refractive index increment was used to analyze the light scattering intensity. The validity of the $M_{\mathrm{w}}$ values was confirmed by the intrinsic viscosity $[\eta]$ in THF at $25^{\circ} \mathrm{C}$ with the known $[\eta]-M_{\mathrm{w}}$ relationship [11]. The weight-average number of repeat unit $N_{\mathrm{w}}$ was thus evaluated to be 117 and 162 for CTPC61K and CTPC84K, respectively.

TCP was purchased from Kishida and used as a solvent without further purification. Each sample which was dried in vacuum at room temperature overnight was weighed with an electronic balance in a glass bottle and an appropriate amount of solvent was added before at least 48 hours prior to use for the following measurements.

\section{Small angle $X$-ray scattering (SAXS) measurements}

SAXS measurements were carried out at the BL40B2 beamline in SPring-8 (Hyogo, Japan) to determine scattering intensities $I(q)$ as a function of the magnitude $q$ of the scattering vector at $25{ }^{\circ} \mathrm{C}$ for pure TCP and four CTPC solutions having different polymer mass concentration $c$ between $1 \times 10^{-2} \mathrm{~g} \mathrm{~cm}^{-3}$ and $5 \times 10^{-2} \mathrm{~g} \mathrm{~cm}^{-3}$. Although the highest concentration is about twice higher than the overlap concentration, good linearity of the Berry square-root plot [32] in the wide concentration range was reported for CTPC in THF [11]. Test solutions or solvent were 
measured in the same quartz capillary cell with a diameter of $2 \mathrm{~mm} \phi$. The obtained two-dimensional image data were analyzed in terms of the circular average method to determine $I(q)$. The wavelength, camera length, and accumulation time were chosen to be $0.10 \mathrm{~nm}, 4000 \mathrm{~mm}$, and $300 \mathrm{~s}$, respectively. The obtained $I(q)$ was normalized by the intensity of the direct beam at the lower end of the capillary to calibrate both intensity of the incident light and the transmittance of X-ray through the cell including solution to determine the excess scattering intensity $\Delta I(q)$ from the solute.

\section{Results and discussion}

The Berry square-root plots [32] for the two CTPC samples in TCP at $25^{\circ} \mathrm{C}$ are displayed in Fig. 2 to extrapolate the scattering intensity to infinite dilution and to $q^{2}=0$. The $z$-average mean-square radius of gyration $\left\langle S^{2}\right\rangle_{z}$ and the second virial coefficient $A_{2}$ are determined from the corresponding initial slopes; note that the optical constant was estimated from the doubly extrapolated value $[c / \Delta I(0)]_{c=0}^{1 / 2}$ with the method reported in ref [33]. The resultant $\left\langle S^{2}\right\rangle_{z}$ and $A_{2}$ data are listed in Table 1 along with the $M_{\mathrm{w}}$ values from the SEC-MALLS measurements. The quite large positive $A_{2}$ indicates that TCP is a good solvent of CTPC.

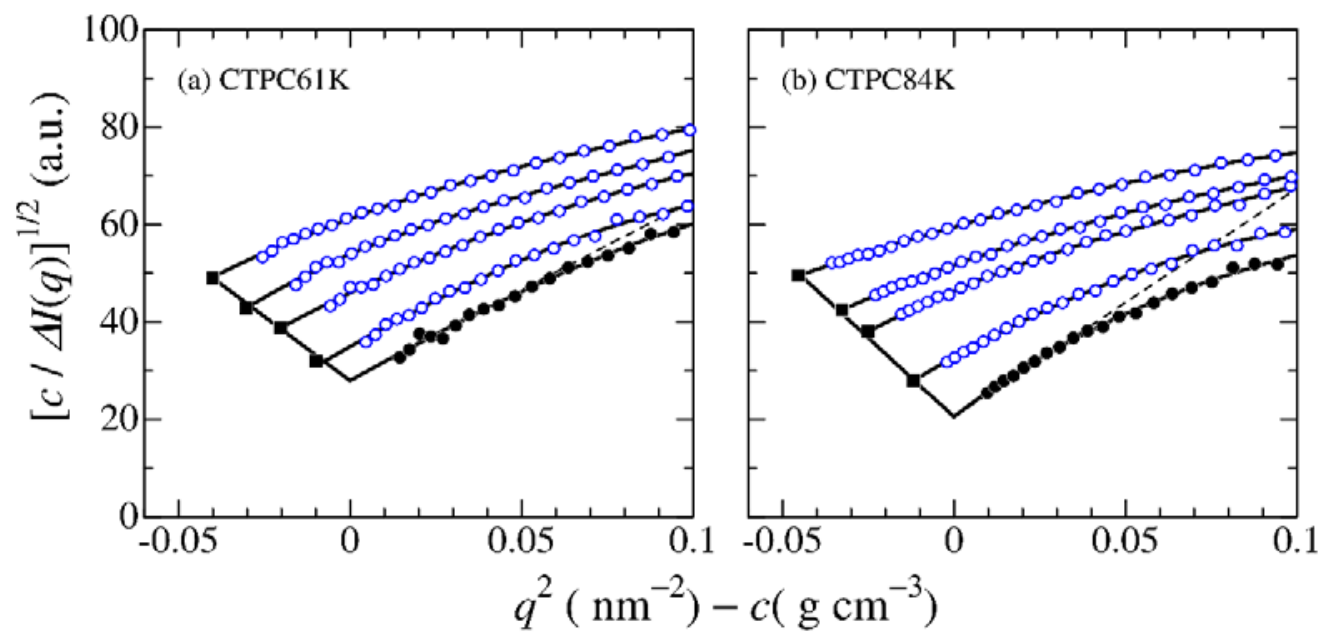

Fig. 2 Square root Zimm plots (Berry plots) for CTPC61K (a) and CTPC84K (b) in TCP at $25^{\circ} \mathrm{C}$. Filled circles and squares, extrapolated values to $c=0$ and $q^{2}=0$, respectively

Table 1 Molecular characteristics of CTPC samples in TCP at $25^{\circ} \mathrm{C}$

\begin{tabular}{ccccc}
\hline Samples & $M_{\mathrm{w}}\left(10^{4} \mathrm{~g} \mathrm{~mol}^{-1}\right)$ & $\left\langle S^{2}\right\rangle_{\mathrm{z}}^{1 / 2}(\mathrm{~nm})$ & $\left\langle S^{2}\right\rangle_{\mathrm{calc}^{1 / 2}}(\mathrm{~nm})^{\mathrm{a}}$ & $A_{2}\left(10^{-4} \mathrm{~mol} \mathrm{~cm}^{3} \mathrm{~g}^{-2}\right)$ \\
\hline CTPC61K & 6.08 & 8.8 & 9.0 & 1.4 \\
CTPC84K & 8.41 & 11.5 & 11.7 & 1.5 \\
\hline
\end{tabular}

${ }^{\text {a }}$ Calculated by eq 3 with the parameters in Table 2 
The particle scattering function $P(q)$ defined as $[\Delta I(q) / \Delta I(0)]_{c=0}$ for each sample was also obtained from the $\Delta I(q)$ data with the extrapolated value $[c / \Delta I(0)]_{c=0}$. Fig. 3 illustrates the reduced Holtzer plots in which the flat plateau is found for each sample at higher $q$ range with an appreciable peak at low $q$. This is a typical feature of the wormlike chain. A slight decrease of $q P(q)$ at the highest $q$ region in the figure may be due to the chain thickness effect. We thus analyzed the $P(q)$ data in terms of the touched-bead wormlike chain with the bead diameter $d$ is expressed as $[34,35]$

$$
P(q)=9\left(\frac{2}{q d}\right)^{6}\left(\sin \frac{q d}{2}-\frac{q d}{2} \cos \frac{q d}{2}\right)^{2} P_{0}(q)
$$

Here, $P_{0}(q)$ is the particle scattering function of the infinitely thin wormlike chain, which is characterized by $\lambda^{-1}$ and the contour length $L$. It is related to the characteristic function $I\left(\lambda^{-1} q ; \lambda t\right)$, that is, the Fourier transform of the distribution function of the end-to-end distance of the wormlike chain of the contour length $t$ and of the Kuhn segment length $\lambda^{-1}$

$$
P_{0}(q)=\frac{2}{L^{2}} \int_{0}^{L}(L-t) I\left(\lambda^{-1} q ; \lambda t\right) d t
$$

In this study we used the approximate expression by Nakamura and Norisuye [36,37] to calculate $I\left(\lambda^{-1} q ; \lambda t\right)$. The three parameters were unequivocally determined by means of the curve fitting procedure. Theoretical curves in Fig. 3 calculated with the obtained parameters in Table 2 successfully reproduce the experimental data. The radii of gyration $\left\langle S^{2}\right\rangle_{\text {calc }}$ calculated from the following Benoit-Doty equation [38]

$$
\left\langle S^{2}\right\rangle_{\text {calc }}=\frac{L}{6 \lambda}-\frac{1}{4 \lambda^{2}}+\frac{1}{4 \lambda^{3} L}-\frac{1}{8 \lambda^{4} L^{2}}[1-\exp (-2 \lambda L)]
$$

with the parameters in Table 2 are listed in the fourth column in Table 1; note that both the chain thickness effect and the excluded volume effect should be negligible on $\left\langle S^{2}\right\rangle_{\text {calc }}$ because of $L>50 d$ [39] and $\lambda L<8$ [40]. The resultant $\left\langle S^{2}\right\rangle_{\text {calc }}$ values are almost equivalent to the corresponding experimental $\left\langle S^{2}\right\rangle_{z}$. Furthermore, the helix pitch per residue $h$ defined as $L / N_{\mathrm{w}}$ was calculated to be $0.51 \pm 0.03 \mathrm{~nm}$, which is substantially the same as those for CTPC in other solvents $[10,11,41]$ and for the crystal structure of CTPC-2-butanone complex [42]. We may thus conclude that both $\left\langle S^{2}\right\rangle_{z}$ and $P(q)$ data for CTPC in TCP are consistently explained by the wormlike chain model. 


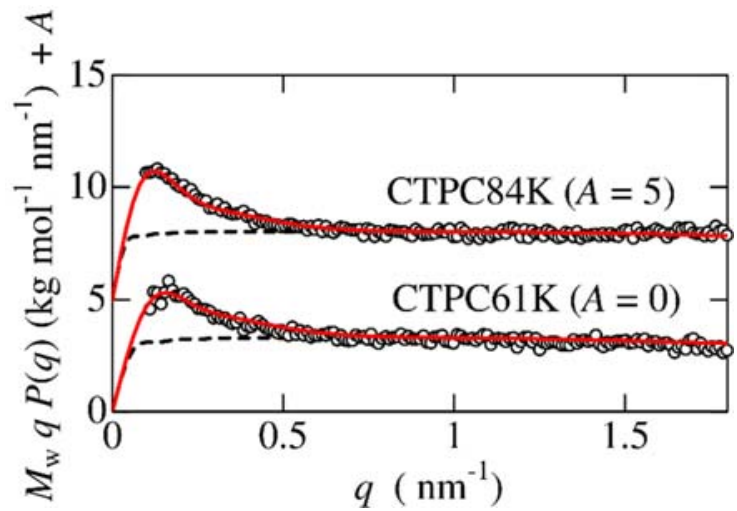

Fig. 3 Reduced Holtzer plots for CTPC in TCP at $25^{\circ} \mathrm{C}$. Solid and dashed curves indicate the theoretical values for the touched bead wormlike chain and the touched bead rigid rod, respectively

Table 2 Wormlike chain parameters for CTPC samples in TCP at $25^{\circ} \mathrm{C}$

\begin{tabular}{lccl}
\hline sample & $L(\mathrm{~nm})$ & $\lambda^{-1}(\mathrm{~nm})$ & $d(\mathrm{~nm})$ \\
\hline CTPC61K & $56 \pm 3$ & $11.5 \pm 0.5$ & $0.9 \pm 0.1$ \\
CTPC84K & $86 \pm 5$ & $11.5 \pm 0.5$ & $0.8 \pm 0.1$
\end{tabular}

The obtained wormlike chain parameters are summarized in Table 3 with previously reported values in the other solvents. If we assume the above mentioned temperature coefficient $\left[\mathrm{d} \ln \lambda^{-1} / \mathrm{d} T \sim-6.5 \times 10^{-3} \mathrm{~K}^{-1}\right][13,22]$, the $\lambda^{-1}$ values in anisol, cyclohexanol, and benzophenone at $25^{\circ} \mathrm{C}$ are estimated to be $10 \mathrm{~nm}, 10 \mathrm{~nm}$, and $14 \mathrm{~nm}$ which are quite close to $11.5 \mathrm{~nm}$. It should be noticed that this estimation might have uncertainty since the temperature coefficient for amylose tris(phenylcarbamate) and polystyrene appreciably depend on the solvent $[43,44]$. In either case, the present $\lambda^{-1}$ in TCP $(11.5 \mathrm{~nm})$ is quite smaller than the other solvents at $25{ }^{\circ} \mathrm{C}$ or $20{ }^{\circ} \mathrm{C}$. We may thus conclude that CTPC in TCP has rather high flexibility in the solvents investigated. 
Table 3 Kuhn segment length $\lambda^{-1}$ for CTPC in various solvents

\begin{tabular}{|c|c|c|c|c|}
\hline solvent & $T\left({ }^{\circ} \mathrm{C}\right)$ & $\lambda^{-1}(\mathrm{~nm})$ & $h(\mathrm{~nm})$ & reference \\
\hline anisole & 94 & 6.2 & $0.52^{\mathrm{a}}$ & {$[6,10]$} \\
\hline cyclohexanol & 73 & 7.6 & $0.52^{\mathrm{a}}$ & {$[6,10]$} \\
\hline benzophenone & 80 & 9.6 & $0.52^{\text {a }}$ & {$[8,10]$} \\
\hline tricresyl phosphate (TCP) & 25 & $11.5 \pm 0.5$ & 0.51 & this work \\
\hline 1-methyl-2-pyrrolidone (NMP) & 25 & 16 & 0.49 & {$[41]$} \\
\hline 1\% LiCl /1,3-dimethyl-2-imidazolidinone (LiCl/DMI) & 25 & 18 & $0.515^{\mathrm{a}}$ & {$[12]$} \\
\hline pyridine & 20 & 20 & $0.52^{\text {a }}$ & {$[4,10]$} \\
\hline tetrahydrofuran (THF) & 25 & $19-24$ & $0.50-0.57$ & [10-12] \\
\hline 1,4-dioxane / methanol 49/51 (v/v) & 25 & 26 & $0.515^{\mathrm{a}}$ & {$[1,5]$} \\
\hline
\end{tabular}

${ }^{\mathrm{a}}$ Assumed.

\section{Conclusion}

The chain stiffness and helix pitch per residue were successfully determined from solution SAXS measurements for CTPC in TCP at $25^{\circ} \mathrm{C}$. The obtained chain stiffness parameter is substantially smaller than those determined around the room temperature and similar to those in some polar solvents when we assume the known temperature coefficient. Significant solvent dependence of the conformational properties indicates that the polymer-solvent interaction is important for the CTPC chain, as also reported for other polysaccharide phenylcarbamate derivatives.

\section{Acknowledgment}

The authors thank Dr. Noboru Ohta (SPring-8) and Dr. Rintaro Takahashi (Kitakyushu Univ.) for SAXS measurements. The synchrotron radiation experiments were performed at the BL40B2 in SPring-8 with the approval of the Japan Synchrotron Radiation Research Institute (JASRI) (Proposal Nos. 2014B1087, 2015A1179, 2015B1100, and 2015B1674). This work was partially supported by JSPS KAKENHI Grant No. 25410130.

\section{References}

1. Burchard W (2008) Light Scattering from Polysaccharides as Soft Materials. In: Borsali R, Pecora R (eds) Soft Matter Characterization. Springer Netherlands, pp 463-603. doi:10.1007/978-1-4020-4465-6_9

2. Hearon WM, Hiatt GD, Fordyce CR (1943) Carbamates of Cellulose and Cellulose Acetate. I. Preparation. J Am Chem Soc 65 (5):829-833. doi:10.1021/ja01245a020

3. Burchard W (1969) Thermodynamic Solution Properties of 3 Polymers Exhibiting Lower Critical Solution Temperature. Polymer 10 (6):467-475. doi:10.1016/0032-3861(69)90055-x

4. Burchard W, Husemann E (1961) Eine Vergleichende Strukturanalyse Von Cellulose-Tricarbanilaten Und Amylose-Tricarbanilaten in Losung. Makromol Chem 44:358-387

5. Burchard W (1965) Uber Die Abweichungen Von Der Idealen Knauelstatistik Bei Amylose- Und

Cellulosetricarbanilat in Einem Theta-Losungsmittel. Makromol Chem 88 (Oct):11-28 
6. Shanbhag VP, Ohman J (1968) Macromolecular Properties of Cellulose Tricarbanilate in Dilute Solutions .4. Unperturbed Dimensions of Cellulose Tricarbanilate from Measurements in Theta Solvents. Arkiv for Kemi 29 (2):163-\&

7. Ohman J (1969) A Light Scattering Study of Cellulose Tricarbanilate in Good Solvents. Arkiv for Kemi 31 (2):125-136

8. Janeschitz-Kriegl H, Burchard W (1968) Flow Birefringence of Short-Chain Molecules - Cellulose

Tricarbanilates in Benzophenone. J Polym Sci A2 6 (12PA):1953-1974. doi:DOI 10.1002/pol.1968.160061202

9. Sutter W, Burchard W (1978) Comparative-Study of Hydrodynamic Properties of Cellulose and Amylose

Tricarbanilates in Dilute-Solutions - Viscosity, Sedimentation and Diffusion Measurements in 1,4-Dioxane in Molecular-Weight Range of 500 Less-Than-or-Equal-to M Less-Than-or-Equal-to 5 . 106. Makromol Chem Macromol Chem Phys 179 (8):1961-1980

10. Daňhelka J, Netopilíak M, Bohdanecký M (1987) Solution properties and chain conformation characteristics of cellulose tricarbanilate. J Polym Sci, Part B: Polym Phys 25 (9):1801-1815. doi:10.1002/polb.1987.090250902

11. Kasabo F, Kanematsu T, Nakagawa T, Sato T, Teramoto A (2000) Solution properties of cellulose tris(phenyl carbamate). 1. Characterization of the conformation and intermolecular interaction. Macromolecules 33 (7):27482756. doi:Doi 10.1021/Ma991443u

12. Yanagisawa M, Isogai A (2005) SEC-MALS-QELS study on the molecular conformation of cellulose in LiCl/amide solutions. Biomacromolecules 6 (3):1258-1265. doi:10.1021/bm049386m

13. Yanai H, Sato T (2006) Local Conformation of the Cellulosic Chain in Solution. Polym J 38 (3):226-233. doi:10.1295/polymj.38.226

14. Yamakawa H, Yoshizaki T (2016) Helical Wormlike Chains in Polymer Solutions, 2nd ed. Springer, Berlin, Germany

15. Nakamura Y, Norisuye T (2012) 2.02 - Polymer Properties in Solutions. In: Editors-in-Chief: Krzysztof M, Martin M (eds) Polymer Science: A Comprehensive Reference. Elsevier, Amsterdam, pp 5-32. doi:10.1016/B978-0444-53349-4.00020-0

16. Norisuye T (1993) Semiflexible Polymers in Dilute-Solution. Prog Polym Sci 18 (3):543-584. doi:Doi $10.1016 / 0079-6700(93) 90017-7$

17. Koyama R, Sato T (2002) Thermodynamic properties of toluene solutions of low molecular weight polystyrenes over wide ranges of concentration. Macromolecules 35 (6):2235-2242. doi:Doi 10.1021/Ma011540z

18. Maeda A, Inoue T, Sato T (2013) Dynamic Segment Size of the Cellulose Chain in an Ionic Liquid. Macromolecules 46 (17):7118-7124. doi:10.1021/ma4010764

19. Jiang XY, Kitamura S, Sato T, Terao K Chain Dimensions and Stiffness of Cellulosic and Amylosic Chains in an Ionic Liquid: Cellulose, Amylose, and an Amylose Carbamate in BmimCl. Macromolecules 50 (10):3979-3984. doi:10.1021/acs.macromol.7b00389

20. Inoue T, Uematsu T, Osaki K (2002) The significance of the Rouse segment: Its concentration dependence. Macromolecules 35 (3):820-826. doi:10.1021/011037m

21. Kratky O, Porod G (1949) Rontgenuntersuchung Geloster Fadenmolekule. Recl Trav Chim Pays-Bas 68 (12):1106-1122

22. Reddy GV, Bohdanecky M (1987) Analysis of the Temperature Quotient of the Intrinsic-Viscosity of Stiff-

Chain Polymers. Macromolecules 20 (6):1393-1396. doi:Doi 10.1021/Ma00172a037

23. Jiang XY, Ryoki A, Terao K (2017) Dimensional and hydrodynamic properties of cellulose tris (alkylcarbamate)s in solution: Side chain dependent conformation in tetrahydrofuran. Polymer 112:152-158. doi:10.1016/j.polymer.2017.02.012

24. Ochiai T, Terao K, Nakamura Y, Yoshikawa C, Sato T (2012) Rigid helical conformation of curdlan tris(phenylcarbamate) in solution. Polymer 53 (18):3946-3950. doi:10.1016/j.polymer.2012.07.004

25. Terao K, Murashima M, Sano Y, Arakawa S, Kitamura S, Norisuye T (2010) Conformational, Dimensional, and Hydrodynamic Properties of Amylose Tris(n-butylcarbamate) in Tetrahydrofuran, Methanol, and Their Mixtures. Macromolecules 43 (2):1061-1068. doi:Doi 10.1021/Ma902200z

26. Sano Y, Terao K, Arakawa S, Ohtoh M, Kitamura S, Norisuye T (2010) Solution properties of amylose tris(nbutylcarbamate). Helical and global conformation in alcohols. Polymer 51 (18):4243-4248.

doi:10.1016/j.polymer.2010.06.048

27. Terao K, Maeda F, Oyamada K, Ochiai T, Kitamura S, Sato T (2012) Side-Chain-Dependent Helical Conformation of Amylose Alkylcarbamates: Amylose Tris(ethylcarbamate) and Amylose Tris(n-hexylcarbamate). J Phys Chem B 116 (42):12714-12720. doi:10.1021/jp307998t 
28. Terao K, Fujii T, Tsuda M, Kitamura S, Norisuye T (2009) Solution Properties of Amylose

Tris(phenylcarbamate): Local Conformation and Chain Stiffness in 1,4-Dioxane and 2-Ethoxyethanol. Polym J 41 (3):201-207. doi:10.1295/polymj.PJ2008233

29. Fujii T, Terao K, Tsuda M, Kitamura S, Norisuye T (2009) Solvent-Dependent Conformation of Amylose Tris(phenylcarbamate) as Deduced from Scattering and Viscosity Data. Biopolymers 91 (9):729-736.

doi:10.1002/bip.21219

30. Tsuda M, Terao K, Nakamura Y, Kita Y, Kitamura S, Sato T (2010) Solution Properties of Amylose Tris(3,5dimethylphenylcarbamate) and Amylose Tris(phenylcarbamate): Side Group and Solvent Dependent Chain Stiffness in Methyl Acetate, 2-Butanone, and 4-Methyl-2-pentanone. Macromolecules 43 (13):5779-5784.

doi:10.1021/ma1006528

31. Lodge TP, Hermann KC, Landry MR (1986) Coil Dimensions of Polystyrenes in Isorefractive Viscous Solvents by Small-Angle Neutron-Scattering. Macromolecules 19 (7):1996-2002. doi:Doi 10.1021/Ma00161a036

32. Berry GC (1966) Thermodynamic and Conformational Properties of Polystyrene .I. Light-Scattering Studies on Dilute Solutions of Linear Polystyrenes. J Chem Phys 44 (12):4550-4564. doi:Doi 10.1063/1.1726673

33. Jiang XY, Terao K, Chung WJ, Naito M (2015) Chain dimensions and intermolecular interactions of polysilanes bearing alkyl side groups over the UV thermochromic temperature. Polymer 68:221-226.

doi:10.1016/j.polymer.2015.05.018

34. Nagasaka K, Yoshizaki T, Shimada J, Yamakawa H (1991) More on the Scattering Function of Helical Wormlike Chains. Macromolecules 24 (4):924-931. doi:Doi 10.1021/Ma00004a018

35. Burchard W, Kajiwara K (1970) The Statistics of Stiff Chain Molecules. I. The Particle Scattering Factor. Proc R Soc London, Ser A 316 (1525):185-199. doi:10.1098/rspa.1970.0074

36. Nakamura Y, Norisuye T (2004) Scattering Function for Wormlike Chains with Finite Thickness. J Polym Sci, Part B: Polym Phys 42 (8):1398-1407. doi:10.1002/polb.20026

37. Nakamura Y, Norisuye T (2008) Brush-Like Polymers. In: Borsali R, Pecora R (eds) Soft Matter

Characterization. Springer Netherlands, pp 235-286. doi:10.1007/978-1-4020-4465-6_5

38. Benoit H, Doty P (1953) Light Scattering from Non-Gaussian Chains. J Phys Chem 57 (9):958-963. doi:10.1021/j150510a025

39. Konishi T, Yoshizaki T, Saito T, Einaga Y, Yamakawa H (1990) Mean-Square Radius of Gyration of Oligostyrenes and Polystyrenes in Dilute-Solutions. Macromolecules 23 (1):290-297. doi:Doi 10.1021/Ma00203a050

40. Norisuye T, Tsuboi A, Teramoto A (1996) Remarks on Excluded-Volume Effects in Semiflexible Polymer Solutions. Polym J 28 (4):357-361. doi:DOI 10.1295/polymj.28.357

41. Norisuye T, Tsuboi A, Sato T, Teramoto A (1997) Solution properties of cellulose Tris(3,5dimethylphenylcarbamate). Macromol Symp 120:65-76. doi:DOI 10.1002/masy.19971200109

42. Zugenmaier P, Vogt U (1983) Structural investigations on cellulose tricarbanilate: Conformation and liquid crystalline behaviour. Makromol Chem 184 (8):1749-1760. doi:10.1002/macp.1983.021840818

43. Asano N, Kitamura S, Terao K (2013) Local Conformation and Intermolecular Interaction of Rigid Ring Polymers Are Not Always the Same as the Linear Analogue: Cyclic Amylose Tris(phenylcarbamate) in Theta Solvents. J Phys Chem B 117 (32):9576-9583. doi:10.1021/jp406607w

44. Terao K, Morihana N, Ichikawa H (2014) Solution SAXS Measurements over a Wide Temperature Range to Determine the Unperturbed Chain Dimensions of Polystyrene and a Cyclic Amylose Derivative. Polym J 46 (3):155-159. doi:Doi 10.1038/Pj.2013.76 